$\underline{\text { Research Article }}$

\title{
Malaria service provision in Manicaland Province, Zimbabwe during the coronavirus pandemic: a cross-sectional survey of health facilities
}

\author{
John Pellegrino ${ }^{1}$, Oscar Tapera², Joseph Mberikunashe ${ }^{3}$, Mufaro Kanyangarara ${ }^{1}$ \\ 1 Department of Epidemiology and Biostatistics, University of South Carolina, Columbia, SC, USA, ${ }^{2}$ SADTAP Health Research Institute, Harare, \\ Zimbabwe, ${ }^{3}$ Ministry of Health and Child Care, Harare, Zimbabwe \\ Keywords: malaria, readiness, health facilities, Covid-19, Zimbabwe \\ https://doi.org/10.29392/001c.31597
}

\section{Journal of Global Health Reports}

Vol. 6, 2022

\begin{abstract}
Background
The ongoing coronavirus disease 2019 (COVID-19) pandemic has disrupted essential health services globally. COVID-19 related disruptions threaten malaria control and elimination as untreated and inappropriately treated malaria cases can lead to excess mortality and impede the COVID-19 response. This study characterized malaria service provision during the pandemic in Manicaland Province, Zimbabwe.
\end{abstract}

\begin{abstract}
Methods
A cross-sectional survey was conducted in randomly selected health facilities in Buhera, Makoni, and Mutare districts in August 2020. Data on the provision of malaria services, the availability of infection prevention control measures and the display of COVID-19 related information, education, and communication (IEC) materials were collected using a standardized questionnaire. Descriptive analyses by facility type, managing authority, and district were used.
\end{abstract}

\section{Results}

A total of 100 health facilities were surveyed and most were publicly managed health centers and clinics. 5\% of health facilities reported testing for COVID-19, 3\% reported COVID-19 related health worker absences and 1\% reported disruptions to any essential health services. About two-thirds (67\%) of facilities displayed COVID-19 related IEC materials and $75 \%$ had infection prevention control measures available. Despite inequities in malaria readiness by managing authority, district, and type of facility, $70 \%$ of facilities were considered "ready" to provide malaria services.

\section{Conclusions}

Malaria services were fairly available with very few COVID-19 related disruptions to essential health services. Maintaining essential malaria services remains crucial in preventing excess malaria morbidity and mortality.

Globally, the coronavirus disease 2019 (COVID-19) pandemic has caused over 173 million cases and 3.7 million deaths to date. ${ }^{1}$ Africa has reported over 3.5 million cases and 87,000 deaths, accounting for about $3 \%$ of the global burden of COVID-19. ${ }^{2}$ These numbers reflect remarkable resilience and adaptivity in the face of the unprecedented challenge of COVID-19. ${ }^{3}$ Nevertheless, these numbers most likely underestimate the true impact of COVID-19 due to limited testing capacity and under-resourced surveillance systems in many African countries. ${ }^{4}$ The pandemic has had devastating impacts on the health sector with far-reaching effects due to inadequate public health infrastructure, limited financial resources, stock-outs of essential medicines, lack of personal protective equipment (PPE), and shortages of health care workers in many African countries. ${ }^{4,5}$ The increasing morbidity and mortality from COVID-19 has placed excess stress on fragile health systems. At least $90 \%$ of countries have reported experiencing disruptions to essential health services, with notable disruptions in neglected tropical diseases, mental health, communicable disease services, and maternal, child and newborn services. ${ }^{6}$ The threat posed by the pandemic on outbreak detection and control for endemic diseases such as HIV, tuberculosis (TB), and malaria continues to be a concern.

In 2019, there were an estimated 229 million malaria cases and 409,000 malaria deaths, of which $94 \%$ of cases 
and deaths occurred in Africa. ${ }^{5}$ These figures represent significant progress towards malaria elimination that has been attributed to the mass distribution of insecticide treated nets (ITNs) and indoor residual spraying (IRS), improved access to rapid diagnostic tests (RDTs) and antimalarial drugs, and strengthened malaria surveillance. ${ }^{7}$ However, the pandemic has the potential to reverse this progress and set the clock back 20 years in global progress towards malaria elimination. ${ }^{8}$ The COVID-19 pandemic has impacted both the uptake and the provision of malaria services. During the pandemic, the demand for services has decreased due to fear of infection, lack of transportation, movement restrictions due to lockdowns, and increased financial constraints limiting the ability to afford transportation and medical costs. ${ }^{9,10}$ Several countries in sub-Saharan Africa (SSA) have reported drops in malaria outpatient attendance following the onset of the COVID-19 pandemic. ${ }^{5}$ Health facilities serve as a point of contact with the health system for malaria diagnosis and treatment and the distribution of ITNs and intermittent preventive treatment in pregnant women (IPTp) and in infants (IPTi). The implementation of the test, treat, and track policy for malaria control relies on the availability of a trained workforce, and in the context of the COVID-19 pandemic, the availability of PPE to ensure infection prevention and control and patient safety. Absenteeism due to illness, fear of infection, restrictions on movement and reassignment of staff to the COVID-19 response have impeded the availability of human resources for health. ${ }^{11,12}$ The production and supply of malaria commodities such as drugs, ITNs, RDT kits, and insecticides for IRS has been delayed and hindered by travel and trade restrictions globally. Reports of limited commodity availability, unreliable logistics systems, and increased lead time for procurements have emerged. ${ }^{13,14}$ The low supply and increased demand for RDTs and antimalarial drugs have driven costs upwards. ${ }^{15}$ The shortage of RDTs and subsequent increased use of presumptive treatment have long-term implications for drug resistance and increases the potential use of substandard or falsified medicines and diagnostics. The provision of essential malaria services has also been negatively impacted by the pandemic with the closure of health facilities, the suspension of outpatient clinics and community health worker (CHW) programs that provide malaria education, diagnosis, and treatment, and the disruptions in IRS and ITN distribution campaigns. ${ }^{16,17}$

In 2020, campaigns for ITNs, IRS, and seasonal malaria chemoprevention (SMC) were suspended or delayed in about $30 \%$ of countries where they had been scheduled. ${ }^{6} \mathrm{~A}$ surge in malaria cases and deaths was observed in several malaria endemic African countries including Zimbabwe as a result of disruptions to malaria services following the start of the COVID-19 pandemic and lockdown restrictions. 5,18 During the Ebola outbreak in 2014-2016, disruptions in essential health services led to an increase in deaths from malaria, HIV/AIDS, and tuberculosis. ${ }^{19}$ For example, in 2014 in Guinea, there were 1,067 malaria-associated deaths compared to 108 deaths in 2013. ${ }^{20}$ The excess malaria deaths during the outbreak exceeded the number of Ebola virus disease (EVD) deaths reported during the outbreak. Initial estimates from the World Health Organization (WHO) estimate that in 2020 alone, globally, there was an excess of 3 million indirect deaths attributable to COVID-19. ${ }^{21}$ Malaria-specific models of the impact of disruptions to ITN distribution and access to antimalarials projected a $22 \%$ increase in malaria cases and almost a doubling of malaria deaths to 769,000 globally. ${ }^{22}$

Manicaland Province, Zimbabwe has the highest burden of malaria cases and the potential to have the greatest disruptions of malaria service due to COVID-19. In 2014, the province reported an incidence of about 116 cases per 1,000 persons with a $48 \%$ RDT positivity rate. ${ }^{23}$ The province has a health facility density of 1.7 per 10,000 population, an inpatient bed density of 16 per 10,000 population, and a health worker density of 6 per 10,000 population. ${ }^{24}$ The public health system consists of 4 levels of care. Quaternary and tertiary hospitals provide specialized care at the central and provincial level respectively, secondary level facilities consist of district hospitals that provide emergency, ambulatory, and inpatient services, and primary level facilities consist of health centers and clinics that serve as the first point of contact with the health system. ${ }^{24,25}$ According to the most recent nationally representative health facility assessment, 99\% of health facilities in Zimbabwe offered malaria diagnosis and treatment services, 97\% had RDT kits, and $96 \%$ had antimalarial drugs in stock. ${ }^{24}$ Based on the availability of trained staff, national guidelines, malaria diagnostics and essential medicines and commodities, 11\% of health facilities were considered "ready" to provide malaria services. ${ }^{24}$

Maintaining essential malaria services remains crucial in preventing excess malaria morbidity and mortality. Nevertheless, there is limited evidence on the availability of malaria services and readiness of health facilities to diagnose and treat malaria during the pandemic. ${ }^{9}$ WHO has urged health care systems to continue essential malaria services during the pandemic to avoid reversing the progress made to improving malaria control globally. ${ }^{8}$ Information on malaria service provision during the pandemic can be used to evaluate the effectiveness of current programs and policies and support targeted strategies to limit the indirect impacts of the pandemic. The overall goal of this study was to assess the current extent of availability of malaria services and infection prevention control measures at health facilities in Manicaland Province, Zimbabwe during the pandemic.

\section{METHODS}

\section{STUDY DESIGN AND SETTING}

The present study was a cross-sectional survey and analysis that was conducted in August 2020 and is a part of a broader study to assess the impact of COVID-19 on malaria prevention and control in Manicaland Province, Zimbabwe. Manicaland Province was selected based on having the highest burden of malaria cases and deaths nationally and the potential to be most affected by COVID-19 related disruptions to malaria services. The present study was con- 
ducted in three randomly selected districts to represent high, moderate, and low malaria transmission areas: $\mathrm{Mu}$ tare, Makoni, and Buhera districts respectively. Based on the 2012 census, Mutare, Makoni, and Buhera districts have a population of $452,863,247,257$, and 247,557 respectively. ${ }^{26}$

A comprehensive listing of health facilities in the selected districts was used to select a random sample of 100 health facilities. The minimum required sample size was 97 based on the assumption that $50 \%$ of facilities had the attribute of interest with an absolute precision of $10 \%$ and 95\% confidence interval. As all hospitals serving the three districts were included, the final sample size was 100 . The hospitals were the Birchenough Bridge Hospital, Sakubva District Hospital, Murambinda Mission Hospital, Mutare Provincial Hospital, and Rusape District Hospital.

A standardized health facility questionnaire was adapted from the Service Availability and Readiness Assessment (SARA) developed by WHO. ${ }^{27}$ The questionnaire assessed the availability of basic amenities, basic equipment, and standard precautions for infection prevention; and the availability of diagnostic capacity, national guidelines, trained staff, and medicines and commodities required for testing and treating malaria. The questionnaire also included questions regarding the availability of COVID-19 testing and the display of IEC materials related to COVID-19. The impact of COVID-19 on staff and service availability was assessed with questions on staff absences and service closures due to the pandemic. Data was collected through direct observation of the facilities and interviews with the facility managers at respective facilities.

\section{STATISTICAL ANALYSIS}

Descriptive analyses by facility type, managing authority, and district were conducted to analyze the differences in the availability of amenities, equipment, infection prevention precautions, malaria resources, and COVID-19 IEC materials. Readiness to provide malaria services was assessed based on the availability of 4 tracer items: national guidelines, trained staff, malaria RDTs, and Artemether-Lumefantrine. Health facilities with all 4 tracer items on the day of assessment were consider ready to provide malaria services. The availability of these 4 items provides a way to compare and measure readiness to provide malaria services between the subgroups of health facilities across the districts. The health facilities were selected via a random sample in order to prevent possible bias.

\section{ETHICAL CONSIDERATIONS}

Ethical approval was obtained from the Medical Research Council of Zimbabwe (MRCZ) prior to the commencement of the study (MRCZ/2633). Permission to conduct heath facility surveys was sought from the Permanent Secretary for Health, the Provincial Medical Director for Manicaland Province and managers of sampled health facilities. In addition, owing to the COVID-19 pandemic at the time of study implementation, the field team adhered to public health measures put in place for limit COVID-19 transmis-

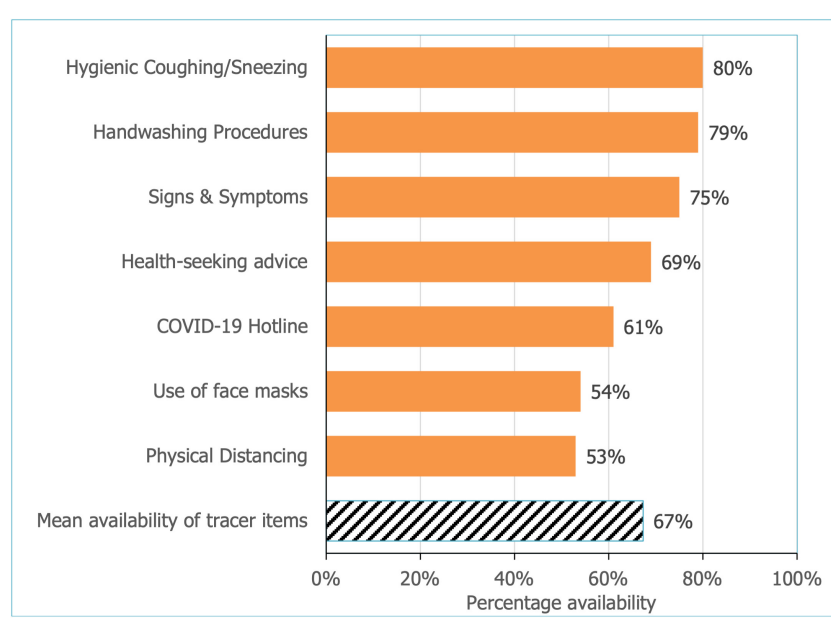

Figure 1. Availability of information, education, and communication (IEC) materials on display at health facilities in Manicaland Province.

sion, including wearing of face masks, social distancing, and frequent handwashing or sanitization.

\section{RESULTS}

The sampled health facilities were evenly distributed across the three districts (Table 1). All sampled health facilities agreed to participate in the health facility assessment. All 100 facilities were observed and given the surveys at the time of the study in August 2020. The majority of health facilities were health centers and clinics (95\%) that were managed by government and public authorities (91\%) (Table 1). 32\% were located in Buhera Distract, $34 \%$ were in Makoni District, and 34\% were in Mutare District. No data was reported as missing.

Only $1 \%$ of health facilities reported the closure of an essential health service due to COVID-19, and only $3 \%$ of health facilities, all of which were located in Makoni District, reported staff absences due to COVID-19. Reasons for staff absences included the lack of PPE as well as a confirmed case of COVID-19 and contact tracing. All hospitals (5\%) reported providing COVID-19 testing in the previous week and the median turnaround time for COVID-19 test results was 2 to 3 days. Rusape District Hospital reported testing the highest number of patients $(n=92)$, followed by Mutare Provincial Hospital $(n=88)$ and Murambinda Mission Hospital $(n=63)$. As health facilities are a source of health information on COVID-19 risks and prevention measures, an assessment of the visual display of IEC materials was conducted. The study indicated that $80 \%$ of the facilities displayed materials regarding hygienic coughing and sneezing, $79 \%$ on handwashing procedures, $75 \%$ on the signs and symptoms of COVID-19, 69\% on health-seeking, $54 \%$ on the use of face masks, $53 \%$ on physical distancing, and $61 \%$ listed the COVID-19 hotline number (Figure 1).

The assessment of the availability of basic equipment and amenities indicated that $93 \%$ of facilities had a thermometer, $91 \%$ had a child scale, $86 \%$ had a stethoscope, $84 \%$ had an adult scale, $84 \%$ had a blood pressure ap- 


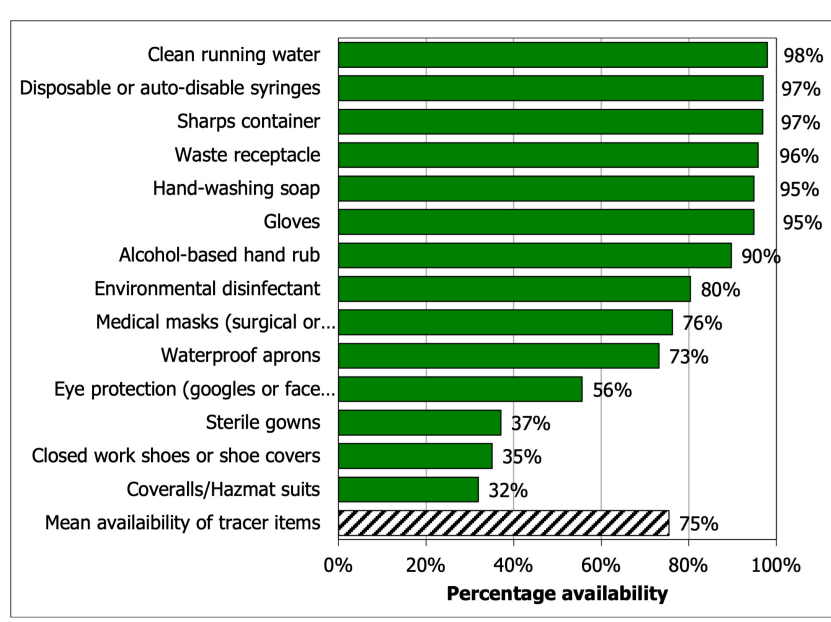

Figure 2. Availability of standard precautions for infection prevention at health facilities in Manicaland Province.

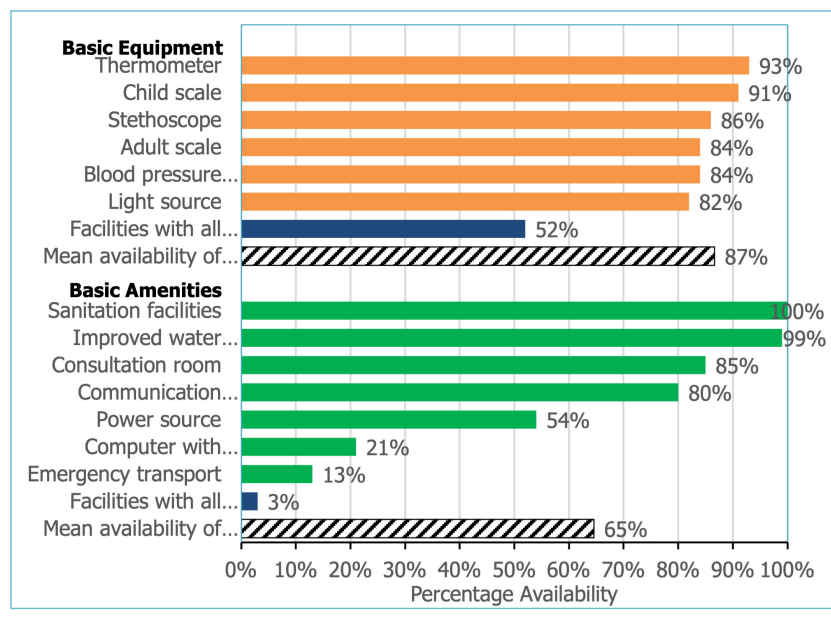

Figure 3. Availability of basic amenities and equipment at health facilities in Manicaland Province.

paratus, and $82 \%$ had a light source (Figure 2). Despite the high availability of basic equipment (mean availability $87 \%$ ), only half (52\%) of health facilities had all 6 basic equipment available. Similarly, basic amenities were available (mean availability 85\%) but availability of all 7 basic amenities was much lower (3\%). Additionally, there was a stark difference in the availability of basic amenities, ranging from $13 \%$ for the availability of emergency transportation to $99 \%$ for the availability of improved water source. For standard precautions for infection prevention, almost all facilities had clean running water (98\%), disposable or auto-disable syringes (97\%), sharps containers (97\%), waste receptacles (96\%), hand-washing soap (95\%), gloves (95\%), and alcohol-based hand rubs (90\%) (Figure 3). However, googles/face shields for eye protection (56\%), sterile gowns (37\%), closed work shoes or shoe covers (35\%), and coveralls or hazmat suits (32\%) were relatively less available.

Almost all health facilities had national guidelines for malaria (91\%), malaria RDT kits (95\%), and first line treatment for uncomplicated malaria (Artemether-Lume- fantrine, 94\%) (Table 1). Additionally, injectable artesunate, oral quinine, doxycycline oral, and clindamycin oral that are used as second line treatment for uncomplicated malaria and treatment of severe malaria were available at $93 \%, 76 \%$, 95\%, and $42 \%$ of health facilities, respectively. Overall, malaria drugs and commodities were more available at health centers and clinics compared to hospitals and at public facilities compared to private facilities.

There were significant differences in the availability of national guidelines (P-value $<0.001)$ and RDTs (Pvalue $=0.01$ ) by managing authority. Public health facilities were more likely to have national guidelines and RDTs compared to privately managed facilities. With the exception of artesunate suppositories and injectable quinine, availability of malaria diagnostics, drugs, guidelines, and staff was higher in Mutare district compared to Buhera and Makoni districts. Overall, $70 \%$ of the health facilities were considered ready to provide malaria services, based on the availability of 4 tracer items. Significantly more health facilities were ready to provide malaria services in Mutare district (91\%) compared to Buhera (63\%) and Makoni (56\%) districts $(\mathrm{P}$-value $<0.001)$.

\section{DISCUSSION}

Despite recent progress towards malaria control and elimination, COVID-19 related disruptions to essential malaria services may reverse these improvements. Maintaining essential malaria services is crucial to preventing excess malaria morbidity and mortality. This study sought to evaluate the impact of the COVID-19 pandemic on the availability of malaria services, infection prevention control measures, and COVID-19 IEC materials in health facilities of Manicaland Province, Zimbabwe. COVID-19 had very little impact on staff absences and the suspension of essential health services. Additionally, most health facilities had standard precautions for infection prevention, though very few had sterile gowns, closed work shoes, and hazmat suits. The study was conducted in August 2020, a few months after the first COVID-19 case was detected. It is likely that the health system did not feel the full brunt of the pandemic in these initial months. Consequently, there were few absences due to COVID-19 and little to no disruptions in the provision of any essential health services. Nevertheless, only 5 facilities, all hospitals reported offering COVID-19 testing. At the time of study implementation, only certain private and public laboratories were approved to test for COVID-19 infection. ${ }^{28}$ Testing was initially done using polymerase chain reaction (PCR), which is typically only available at tertiary hospitals and private laboratories. ${ }^{28,29}$ Since August 2020 when the study was implemented, the Ministry of Health and Child Care in Zimbabwe has trained staff and distributed RDTs to health clinics to increase COVID-19 testing rates. ${ }^{29}$ Given the overlapping clinical symptoms of COVID-19 and malaria, diagnosis is a critical issue. Additionally, co-infection with malaria and COVID-19 may lead to more severe complications and increase the risk of death. ${ }^{30} \mathrm{~A}$ double-pronged approach that 
Table 1. Availability of malaria services at health facilities in Manicaland Province.

\begin{tabular}{|c|c|c|c|c|c|c|c|c|c|c|c|c|c|c|}
\hline & $\begin{array}{l}\text { Number } \\
\text { of } \\
\text { health } \\
\text { facilities }\end{array}$ & $\begin{array}{l}\text { National } \\
\text { guidelines } \\
\text { for } \\
\text { malaria }\end{array}$ & $\begin{array}{l}\text { Trained } \\
\text { Staff }\end{array}$ & $\begin{array}{l}\text { Malaria } \\
\text { RDTs }\end{array}$ & $\begin{array}{l}\text { Stock- } \\
\text { out of } \\
\text { malaria } \\
\text { RDTs }\end{array}$ & $\begin{array}{l}\text { Artemether- } \\
\text { Lumefantrine }\end{array}$ & $\begin{array}{l}\text { Injectable } \\
\text { artesunate }\end{array}$ & $\begin{array}{l}\text { artesunate } \\
\text { suppositories/ } \\
\text { rectal } \\
\text { artesunate }\end{array}$ & $\begin{array}{l}\text { Sulfadoxine + } \\
\text { Pyrimethamine } \\
\text { (SP) }\end{array}$ & $\begin{array}{l}\text { Oral } \\
\text { quinine }\end{array}$ & $\begin{array}{l}\text { Injectable } \\
\text { quinine }\end{array}$ & $\begin{array}{l}\text { Oral } \\
\text { doxycycline }\end{array}$ & $\begin{array}{l}\text { Oral } \\
\text { clindamycin }\end{array}$ & $\begin{array}{l}\text { Facilities } \\
\text { with all } \\
\text { Tracer } \\
\text { Items* }\end{array}$ \\
\hline \multicolumn{15}{|l|}{ District } \\
\hline Buhera & 32 & $88 \%$ & $81 \%$ & $97 \%$ & $3 \%$ & $91 \%$ & $91 \%$ & $38 \%$ & $94 \%$ & $63 \%$ & $9 \%$ & $91 \%$ & $41 \%$ & $63 \%$ \\
\hline Makoni & 34 & $91 \%$ & $71 \%$ & $88 \%$ & $0 \%$ & $91 \%$ & $88 \%$ & $44 \%$ & $79 \%$ & $74 \%$ & $18 \%$ & $94 \%$ & $38 \%$ & $56 \%$ \\
\hline $\begin{array}{l}\text { Mutare } \\
\text { Rural }\end{array}$ & 34 & $94 \%$ & $97 \%$ & $100 \%$ & $3 \%$ & $100 \%$ & $100 \%$ & $38 \%$ & $94 \%$ & $91 \%$ & $6 \%$ & $100 \%$ & $47 \%$ & $91 \%$ \\
\hline P-Value & & 0.6 & 0.01 & 0.07 & 0.6 & 0.2 & 0.1 & 0.8 & 0.09 & 0.02 & 0.3 & 0.2 & 0.8 & 0.003 \\
\hline \multicolumn{15}{|c|}{ Facility type } \\
\hline Hospital & 5 & $60 \%$ & $100 \%$ & $80 \%$ & $0 \%$ & $60 \%$ & $60 \%$ & $20 \%$ & $60 \%$ & $60 \%$ & $20 \%$ & $60 \%$ & $20 \%$ & $40 \%$ \\
\hline $\begin{array}{l}\text { Health } \\
\text { Center/ } \\
\text { Clinic } \\
\end{array}$ & 95 & $93 \%$ & $82 \%$ & $96 \%$ & $2 \%$ & $96 \%$ & $95 \%$ & $41 \%$ & $91 \%$ & $77 \%$ & $11 \%$ & $97 \%$ & $43 \%$ & $72 \%$ \\
\hline P-Value & & 0.01 & 0.6 & 0.2 & 0.9 & 0.001 & 0.003 & 0.6 & 0.04 & 0.4 & 0.01 & $<0.001$ & 0.5 & 0.11 \\
\hline \multicolumn{15}{|c|}{ Managing authority } \\
\hline Public & 91 & $95 \%$ & $82 \%$ & $97 \%$ & $2 \%$ & $95 \%$ & $95 \%$ & $40 \%$ & $89 \%$ & $77 \%$ & $11 \%$ & $97 \%$ & $43 \%$ & $73 \%$ \\
\hline Private & 9 & $56 \%$ & $89 \%$ & $78 \%$ & $0 \%$ & $89 \%$ & $78 \%$ & $44 \%$ & $89 \%$ & $67 \%$ & $11 \%$ & $78 \%$ & $33 \%$ & $44 \%$ \\
\hline P-Value & & $<0.001$ & 0.4 & 0.01 & 1 & 0.7 & 0.07 & 0.6 & 1 & 0.7 & 0.9 & 0.01 & 0.8 & 0.2 \\
\hline Total & 100 & $91 \%$ & $83 \%$ & $95 \%$ & $2 \%$ & $94 \%$ & $93 \%$ & $40 \%$ & $89 \%$ & $76 \%$ & $11 \%$ & $95 \%$ & $42 \%$ & $70 \%$ \\
\hline
\end{tabular}

*The 4 tracer items include: national guidelines, trained staff, malaria RDTs, and Artemether-Lumefantrine. RDTs= rapid diagnostic tests 
addresses the direct and indirect consequences of the COVID-19 pandemic on malaria is therefore needed.

The present study also indicated that $70 \%$ of facilities in Manicaland Province were ready to provide malaria services based on the availability of national guidelines, RDTs, trained staff, and Artemether-Lumefantrine. By contrast, in 2015 , only $11 \%$ of health facilities in Zimbabwe were ready to provide malaria services. ${ }^{24}$ While no inferences can be made about the impact of COVID-19 on the provision of malaria services, the findings suggest that most health facilities had the capacity to provide malaria diagnosis and treatment. Nevertheless, a recent assessment of routine malaria surveillance in Zimbabwe indicated excess malaria morbidity and mortality following the onset of the pandemic. ${ }^{18}$ These findings coupled with the malaria service provision results of the present study suggest that while malaria services were maintained during the pandemic, there were increases in malaria incidence and mortality which could reflect decreased service utilization. Recent reports show declines in general outpatient attendance and malaria outpatient attendance in 2020 compared to 2019 in Zimbabwe and other malaria endemic countries. ${ }^{9}$

The study also found apparent differences in malaria service readiness between districts; more health facilities in Mutare district were ready to provide malaria services than in Makoni and Buhera districts. Malaria readiness was lowest in Buhera district which has the lowest malaria transmission and lower availability of trained staff and national malaria guidelines. Previous studies have shown that malaria service provision in sub-Saharan Africa is generally focused in endemic areas where the transmission rates of malaria are highest. ${ }^{31-33}$ Therefore, it is unsurprising that Mutare had a greater availability of malaria services. In addition, Buhera district is more rural than the other districts. Rural areas tend to have lower-level health facilities that are not as prepared to provide essential malaria services. ${ }^{24}$ The study also discovered that the public sector was more prepared to provide malaria services than the private sector, in part because of shortages of malaria RDTs at private health facilities. Several other studies in Africa have similarly found that the private sector lacked access to malaria diagnostic testing due to national regulatory frameworks hindering the procurement of RDTs by private health facilities. ${ }^{34}$ Private health facilities need to address the shortage of RDTs to fortify their malaria testing capacity and reduce the burden of malaria during future waves of epidemics and pandemics,

A few limitations of the study should be considered. The study was a cross-sectional study providing a snapshot of malaria service provision and the impact of the pandemic at one point in time. Casual inferences cannot be made. The study was conducted in August 2020, very early in the pandemic, and the findings may not reflect the full impact of the pandemic. While the findings are representative of Manicaland Province, the findings may not be generalizable to other parts of Zimbabwe.

\section{CONCLUSIONS}

Limitations notwithstanding, the study found that there were very few gaps in malaria service provision during the pandemic at the time of the study; most of the health facilities in Manicaland Province had the capacity and infection prevention control measures in place to appropriately provide malaria services amid the pandemic. With the commencement of the rainy season in many malaria-endemic countries, the emergence of SARS-CoV-2 variants and a pending wave of COVID-19 in Africa, governments should continue to support health systems strengthening and resilience building to mitigate the impact of COVID-19 and future crises. ${ }^{35}$ It remains essential that healthcare facilities provide malaria services while ensuring infection prevention to mitigate the potential indirect effects of the COVID-19 pandemic.

\section{ACKNOWLEDGEMENTS}

The authors would like to thank the staff members at participating health facilities.

\section{FUNDING}

This research was funded by University of South Carolina start-up funds awarded to Dr. Kanyangarara.

\section{AUTHORSHIP CONTRIBUTIONS}

JP contributed to data analysis and interpretation and drafting of the manuscript. AM contributed to data analysis and revised the manuscript critically for important intellectual content. OT conceived the study, contributed to the study design, data collection, and revised the manuscript critically for important intellectual content. JM contributed to the study design and revised the manuscript critically for important intellectual content. MK conceived the study, contributed to the study design, data analysis, and interpretation and drafting of the manuscript. All authors read and approved the final version of the manuscript.

\section{COMPETING INTERESTS}

The authors completed the Unified Competing Interest form at http://www.icmje.org/disclosure-of-interest/ (available upon request from the corresponding author), and declare no conflicts of interest.

\section{CORRESPONDENCE TO:}

Mufaro Kanyangarara, Department of Epidemiology and Biostatistics, Arnold School of Public Health, University of South Carolina, 915 Greene Street, Columbia, SC 29201. mufaro@mailbox.sc.edu

Submitted: November 17, 2021 GMT, Accepted: December 28, 2021 GMT 
This is an open-access article distributed under the terms of the Creative Commons Attribution 4.0 International License (CCBY-4.0). View this license's legal deed at http://creativecommons.org/licenses/by/4.0 and legal code at http://creativecommons.org/licenses/by/4.0/legalcode for more information. 


\section{REFERENCES}

1. WHO Coronavirus (COVID-19) Dashboard I WHO Coronavirus (COVID-19) Dashboard With Vaccination Data. Accessed June 3, 2021. https://covid19.who.int/

2. Africa Center for Disease Control and Prevention. Outbreak Brief 73: Coronavirus Disease 2019 (COVID-19) Pandemic. African Union; 2021.

3. Blanton RE, Mock NB, Hiruy HN, et al. African resources and the promise of resilience against COVID-19. American Journal of Tropical Medicine and Hygiene. 2020;103(2):539-541. doi:10.4269/ajtmh.2 $\underline{0-0470}$

4. Zawawi A, Alghanmi M, Alsaady I, Gattan H, Zakai $\mathrm{H}$, Couper K. The impact of COVID-19 pandemic on malaria elimination. Parasite epidemiology and control. 2020;11:e00187. doi:10.1016/j.parepi.2020.e0 $\underline{0187}$

5. World Health Organization. World Malaria Report 2020: 20 Years of Global Progress \& Challenges. World Health Organization; 2020.

6. World Health Organization. Pulse Survey on Continuity of Essential Health Services during the COVID-19 Pandemic: Interim Report, 27 August 2020. World Health Organization; 2020.

7. Weiss DJ, Bertozzi-Villa A, Rumisha SF, et al. Indirect effects of the COVID-19 pandemic on malaria intervention coverage, morbidity, and mortality in Africa: a geospatial modelling analysis. The Lancet Infectious Diseases. 2021;21(1):59-69. do i:10.1016/s1473-3099(20)30700-3

8. Thornton J. Covid-19: Keep essential malaria services going during pandemic, urges WHO. BMJ (Clinical research ed). 2020;369:m1637. doi:10.1136/b mi.m1637

9. The Global Fund, Unite to Fight. The Impact of COVID-19 on HIV, TB and Malaria Services and Systems for Health: A Snapshot from 502 Health Facilities across Africa and Asia. The Global Fund; 2021.

10. Roberton T, Carter ED, Chou VB, et al. Early estimates of the indirect effects of the COVID-19 pandemic on maternal and child mortality in lowincome and middle-income countries: a modelling study. The Lancet Global Health. 2020;8(7):e901-e908. doi:10.1016/s2214-109x(20)30229-1
11. Zewudie A, Regasa T, Kebede O, et al. Healthcare professionals' willingness and preparedness to work during COVID-19 in selected hospitals of southwest Ethiopia. Risk Management and Healthcare Policy. 2021;14:391-404. doi:10.2147/rmhp.s289343

12. Afulani PA, Gyamerah AO, Nutor JJ, et al. Inadequate preparedness for response to COVID-19 is associated with stress and burnout among healthcare workers in Ghana. PLoS ONE. 2021;16(4):e0250294. $\underline{\mathrm{d}}$ oi:10.1371/journal.pone.0250294

13. Aborode AT, David KB, Uwishema O, et al. Fighting covid-19 at the expense of malaria in Africa: The consequences and policy options. American Journal of Tropical Medicine and Hygiene. 2021;104(1):26-29. doi:10.4269/ajtmh.20-1181

14. Guerra CA, Tresor Donfack O, Motobe Vaz L, et al. Malaria vector control in sub-Saharan Africa in the time of COVID-19: no room for complacency. BMJ global health. 2020;5(9):e003880. doi:10.1136/bmig $\underline{\mathrm{h}-2020-003880}$

15. Sefah IA, Ogunleye OO, Essah DO, et al. Rapid Assessment of the Potential Paucity and Price Increases for Suggested Medicines and Protection Equipment for COVID-19 Across Developing Countries With a Particular Focus on Africa and the Implications. Frontiers in Pharmacology. 2021;11:2055. doi:10.3389/fphar.2020.588106

16. Diptyanusa A, Zablon KN. Addressing budget reduction and reallocation on health-related resources during COVID-19 pandemic in malariaendemic countries. Malaria Journal. 2020;19(1):1-4. $\underline{\mathrm{d}}$ oi:10.1186/s12936-020-03488-y

17. World Health Organization. Second Round of the National Pulse Survey on Continuity of Essential Health Services during the COVID-19 Pandemic: JanuaryMarch 2021. World Health Organization; 2021.

18. Gavi S, Tapera O, Mberikunashe J, Kanyangarara M. Malaria incidence and mortality in Zimbabwe during the COVID-19 pandemic: analysis of routine surveillance data. Malaria journal. 2021;20(1):233. do $\mathrm{i}: 10.1186 / \mathrm{s} 12936-021-03770-7$

19. Parpia AS, Ndeffo-Mbah ML, Wenzel NS, Galvani AP. Effects of response to 2014-2015 ebola outbreak on deaths from malaria, HIV/AIDS, and tuberculosis, West Africa. Emerging Infectious Diseases. 2016;22(3):433-441. doi:10.3201/eid2203.150977 
20. Wang J, Xu C, Wong YK, et al. Preparedness is essential for malaria-endemic regions during the COVID-19 pandemic. The Lancet.

2020;395(10230):1094-1096. doi:10.1016/s0140-673 $\underline{6(20) 30561-4}$

21. The true death toll of COVID-19: estimating global excess mortality. Accessed June 16, 2021. http s://www.who.int/data/stories/the-true-death-toll-ofcovid-19-estimating-global-excess-mortality

22. Rogerson SJ, Beeson JG, Laman M, et al. Identifying and combating the impacts of COVID-19 on malaria. BMC medicine. 2020;18(1):239. doi:10.118 6/s12916-020-01710-X

23. Mutsigiri F, Mafaune PT, Mungati M, et al. Malaria morbidity and mortality trends in Manicaland province, Zimbabwe, 2005-2014. The Pan African medical journal. 2017;27:30. doi:10.11604/pa mi.2017.27.30.11130

24. Ministry of Health \& Child Care of Zimbabwe. Zimbabwe Service Availability and Readiness Assessment 2015 Report. Government of Zimbabwe; 2015 .

25. Ministry of Health \& Child Care of Zimbabwe. Zimbabwe National Health Financing Policy: "Resourcing Pathway to Universal Health Coverage." Government of Zimbabwe; 2016.

26. Zimbabwe National Statistics Agency, United Nations Population Fund. Manicaland Province District Population Projections Report. Government of Zimbabwe; 2020.

27. World Health Organization. Service Availability and Readiness Assessment (SARA): An Annual Monitoring System for Service Delivery: Reference Manual. World Health Organization; 2013.
28. COVID-19 Information |U.S. Embassy in Zimbabwe. Accessed June 20, 2021. https://zw.usembassy.gov/cov id-19-information-2/

29. Rapid tests up Zimbabwe's COVID-19 diagnosis | WHO | Regional Office for Africa. Accessed June 20, 2021. https://www.afro.who.int/news/rapid-tests-zim babwes-covid-19-diagnosis

30. Wilairatana P, Masangkay FR, Kotepui KU, Milanez GDJ, Kotepui M. Prevalence and characteristics of malaria among COVID-19 individuals: A systematic review, meta-analysis, and analysis of case reports. PLOS Neglected Tropical Diseases. 2021;15(10):e0009766. doi:10.1371/journa l.pntd.0009766

31. United States Agency for International Development. Assessment of the Zimbabwe Assistance Program in Malaria. United States Government; 2020.

32. Lee EH, Olsen CH, Koehlmoos T, et al. A crosssectional study of malaria endemicity and health system readiness to deliver services in Kenya, Namibia and Senegal. Health Policy and Planning. 2017;32(suppl_3):iii75-iii87. doi:10.1093/heapol/czx1 $\underline{14}$

33. Taylor C, Linn A, Wang W, Florey L, Moussa H. Examination of malaria service utilization and service provision: An analysis of DHS and SPA data from Malawi, Senegal, and Tanzania. Malaria Journal. 2019;18(1):1-13. doi:10.1186/s12936-019-2892-x

34. Kaula H, Buyungo P, Opigo J. Private sector role, readiness and performance for malaria case management in Uganda, 2015. Malaria Journal. 2017;16(1):219. doi:10.1186/s12936-017-1824-X

35. Heuschen AK, Lu G, Razum O, et al. Public health-relevant consequences of the COVID-19 pandemic on malaria in sub-Saharan Africa: A scoping review. medRxiv. Published online August 11, 2021. 